\title{
Japanese subpopulation analysis of MONARCH 2: phase 3 study of abemaciclib plus fulvestrant for treatment of hormone receptor-positive, human epidermal growth factor receptor 2-negative breast cancer that progressed on endocrine therapy
}

\author{
Kenichi Inoue ${ }^{1} \cdot$ Norikazu Masuda $^{2} \cdot$ Hiroji Iwata $^{3} \cdot$ Masato Takahashi $^{4} \cdot$ Yoshinori Ito $^{5} \cdot$ Yasuo Miyoshi $^{6}$. \\ Takahiro Nakayama $^{7} \cdot$ Hirofumi Mukai $^{8} \cdot$ Jan-Stefan van der Walt ${ }^{9} \cdot$ Joji Mori $^{10} \cdot$ Sachi Sakaguchi $^{10}$. \\ Tsutomu Kawaguchi $^{10} \cdot$ Yoshinori Tanizawa $^{10}$. Antonio Llombart-Cussac ${ }^{11,12} \cdot$ George W. Sledge Jr $^{13}$. \\ Masakazu Toi ${ }^{14}$
}

Received: 21 December 2020 / Accepted: 11 March 2021 / Published online: 1 April 2021

(c) The Author(s) 2021

\begin{abstract}
Background This was a Japanese subpopulation analysis of MONARCH 2, a double-blind, randomized, placebo-controlled, phase 3 study of abemaciclib plus fulvestrant in patients with hormone receptor-positive, human epidermal growth factor receptor 2-negative advanced breast cancer (ABC).

Methods Eligible women had progressed on (neo)adjuvant endocrine therapy (ET), $\leq 12$ months from end of adjuvant ET, or on first-line ET for ABC, and had not received chemotherapy for ABC. Patients were randomized 2:1 to receive abemaciclib or placebo plus fulvestrant. The primary endpoint was progression-free survival (PFS). Secondary endpoints included overall survival (OS), pharmacokinetics (PK), health-related quality of life (HRQoL), and safety.

Results In Japan, 95 patients were randomized (abemaciclib, $n=64$; placebo, $n=31$ ). At final PFS analysis (February 14, 2017), median PFS was 21.2 and 14.3 months, respectively, in the abemaciclib and placebo groups (hazard ratio: 0.672 ; 95\% confidence interval: 0.380-1.189). Abemaciclib had a higher objective response rate (37.5\%) than placebo (12.9\%). PK and safety profiles for Japanese patients were consistent with those of the overall population, without clinically meaningful differences across most HRQoL dimensions evaluated. The most frequent adverse events in the abemaciclib versus placebo groups were diarrhea (95.2 versus $25.8 \%$ ), neutropenia (79.4 versus 0\%), and leukopenia (66.7 versus $0 \%$ ). At a second data cutoff (June 20, 2019), median OS was not reached with abemaciclib and 47.3 months with placebo (hazard ratio: 0.755 ; 95\% confidence interval: 0.390-1.463).

Conclusions Results of the Japanese subpopulation were consistent with the improved clinical outcomes and manageable safety profile observed in the overall population.

Clinical trial registration NCT02107703; U.S. National Library of Medicine: https://clinicaltrials.gov/ct2/show/NCT02 107703.
\end{abstract}

Keywords Abemaciclib · Breast cancer · Cyclin-dependent kinase 4 and 6 inhibitor

\section{Introduction}

Breast cancer is the second leading cause of cancer mortality in women globally [1]. Women diagnosed with hormone receptor-positive $(\mathrm{HR}+)$, human epidermal growth

Masakazu Toi

toi@ kuhp.kyoto-u.ac.jp

Extended author information available on the last page of the article factor receptor 2-negative (HER2-) breast cancer are typically treated with endocrine therapy (ET), but intrinsic and acquired ET resistance are common issues in patients with advanced or metastatic breast cancer [2, 3].The cyclin D pathway is an important target for overcoming mechanisms of ET resistance [4]. Targeting this pathway with cyclindependent kinase 4 and 6 (CDK4 and CDK6) inhibitors in combination with ET results in significant improvement in clinical outcomes over ET alone [5-15], with CDK4 
and CDK6 inhibitor/ET combination therapy emerging as the new standard of care in the treatment of HR+, HER2 - advanced breast cancer (ABC) [16].

Abemaciclib is a selective small molecule inhibitor of CDK4 and CDK6 orally administered on a continuous twice-daily dosing regimen [17-19]. In preclinical cancer models, continuous inhibition of CDK4 and CDK6 by abemaciclib led to cell cycle arrest and death of cancer cells [18, 20]. Within the abemaciclib clinical development program, MONARCH 2 was a randomized, double-blind, global, phase 3 study of abemaciclib in combination with fulvestrant in women with HR+, HER2- ABC whose disease had progressed while receiving prior ET. In the intent-to-treat (ITT) population, abemaciclib plus fulvestrant significantly extended both progression-free survival (PFS; median: 16.4 versus 9.3 months, hazard ratio [HR]: $0.553,95 \%$ confidence interval $[\mathrm{CI}]: 0.449-0.681, p<0.001)$ and overall survival (OS; median: 46.7 versus 37.3 months, HR: 0.757 ; 95\% CI: $0.606-0.945 ; p=0.01$ ) compared with placebo plus fulvestrant $[12,13]$.

Based on the findings of the global clinical development program, abemaciclib was approved for use in Japan in September 2018 in combination with ET for the treatment of HR+, HER2- ABC. However, ethnicity and country-specific differences in clinical practice can influence response to breast cancer treatment $[21,22]$, and potential interethnic differences in response to abemaciclib have not been extensively studied. The objective of the current analysis was to assess efficacy and safety outcomes in Japanese breast cancer patients within the MONARCH 2 population. Here, we report PFS, safety, patient-reported health-related quality of life (HRQoL), and pharmacokinetic (PK) outcomes of the Japanese subpopulation of MONARCH 2 at the time of the final PFS analysis. In addition, we report OS, time to chemotherapy (TTC), chemotherapy-free survival (CFS), and updated PFS and safety of the Japanese subpopulation at a second data cutoff date, 27 months following the final PFS analysis.

\section{Patients and methods}

\section{Study design and patients}

This analysis was conducted on patients enrolled at study sites in Japan for the global MONARCH 2 study (NCT02107703), a randomized, double-blind, placebocontrolled study of abemaciclib plus fulvestrant in women with ABC (Online Resource 1). Study design and methods for MONARCH 2 have previously been published [12, 13, 23]. Patients were required to have HR+, HER2- inoperable locally advanced or metastatic breast cancer that progressed on neoadjuvant/adjuvant ET, $\leq 12$ months from end of adjuvant ET, or on first-line ET for $\mathrm{ABC}$ and who had not received chemotherapy for advanced disease. Patients were excluded if they had prior treatment with fulvestrant, everolimus, or CDK4 and CDK6 inhibitors, or had visceral crisis or evidence/history of central nervous system metastasis.

The study was conducted in accordance with the 1964 Declaration of Helsinki and its later amendments and the relevant laws and regulations in Japan. The protocol was reviewed by ethical and institutional review boards at the participating institutions. Informed consent was obtained from all individual participants in the study.

\section{Treatments and procedures}

Patients were randomized 2:1 to receive abemaciclib plus fulvestrant or placebo plus fulvestrant, stratified by metastatic site and resistance to prior ET (primary or secondary, as defined in European Society for Medical Oncology guidelines [24, 25]). Fulvestrant (500 mg, per label) was administered by intramuscular injection on Days 1 and 15 of the first cycle and on Day 1 of subsequent cycles (every 28 days). Abemaciclib ( $200 \mathrm{mg}$; reduced to $150 \mathrm{mg}$ following a protocol amendment [13]) or placebo was administered orally twice daily. Permitted dose adjustments in MONARCH 2 were previously described [13]. Treatment continued until progressive disease (PD), death, or patient withdrawal. Crossover between treatment groups was not permitted. Response was determined by investigators for all patients whose disease was evaluable using Response Evaluation Criteria In Solid Tumors (RECIST) version (v) 1.1 [26].

\section{Outcomes}

Efficacy analyses included all patients in the ITT Japanese subpopulation, regardless of starting dose for abemaciclib. The primary efficacy endpoint was the comparison of PFS between treatment groups. Secondary efficacy outcomes included OS, objective response rate (ORR; the proportion of patients with a best response of complete [CR] or partial response $[\mathrm{PR}]$ ), disease control rate (DCR; $\mathrm{CR}+\mathrm{PR}+$ stable disease), and clinical benefit rate (CBR; $\mathrm{CR}+\mathrm{PR}+$ stable disease $\geq 6$ months).

Additional secondary endpoints included safety, PK, and HRQoL, including global health status, functioning, and symptoms. Safety was evaluated in all patients who received at least one dose of study treatment, with treatment-emergent adverse events (TEAEs) summarized using Medical Dictionary for Regulatory Activities (MedDRA) v.19.1 terminology and graded based on the National Cancer Institute Common Terminology Criteria for Adverse Events (CTCAE) v.4.0. PK analyses were conducted on patients in the safety population who had plasma samples collected, which were 
obtained at prescheduled times on Days 1 ( $2-4$ h postdose) and 15 (4-7.0 h postdose) of Cycle 1 and on Day 1 of Cycle 2 (predose and $3.0 \mathrm{~h}$ postdose) and Cycle 3 (predose). The concentration of abemaciclib in plasma was measured using validated liquid chromatography/tandem mass spectrometry assays.

HRQoL analyses included all patients who completed baseline assessment plus at least one post-baseline assessment, as described [23]. Data were collected using the European Organization for Research and Treatment of Cancer Quality of Life Questionnaire-Core 30 (EORTC QLQ-C30) to assess cancer-related QoL [27] and the EORTC QLQBreast Cancer module (EORTC QLQ-BR23) to assess breast cancer-specific QoL [28].

Exploratory endpoints included TTC, CFS, and time to sustained deterioration (TTSD) on the EORTC-QLQ-C30 and QLQ-BR23.

\section{Statistical analyses}

Analyses were conducted at two database locks. PFS, safety, HRQoL, TTSD on HRQoL measures, and PK outcomes are reported at the data cutoff date of February 14, 2017. OS and exploratory endpoints (TTC, CFS) are reported at a second data cutoff date of June 20,2019, at which time updates on PFS and safety are also provided. Statistical methods for MONARCH 2 have been previously described [12, 13, 23]. For this subpopulation analysis, $p$ values for comparisons between treatments are not reported due to the limited sample size. Interim and final PFS and OS analyses were preplanned (Online Resource 1) [12, 13]. PFS, OS, CFS, and TTC were estimated using the Kaplan-Meier method [29], and a Cox proportional hazard model was used to estimate the HRs and corresponding 95\% CIs. Normal approximation was used to estimate $95 \%$ CIs for ORR, DCR, CBR, and the difference in 36-month PFS rates between the treatment groups.

For EORTC-QLQ-C30 and QLQ-BR23, a score ranging from 0 to 100 was calculated for each scale, with a higher score representing more severe symptoms for symptom scales and better health condition for global health status and functioning scales. Change from baseline over the entire treatment course was assessed using mixed effects-repeated measures models including all data and cycles for which at least $25 \%$ of patients completed questionnaires in both study groups. Post hoc analyses investigated TTSD using Cox proportional hazard models. A minimally important difference (MID) of $\geq 10$-points [30], considered herein as a clinically meaningful difference, was utilized for TTSD and change from baseline analyses. TTSD for each scale was defined as the time from randomization to the time at which $\mathrm{a} \geq 10$-point worsening compared with a patient's baseline score was observed, followed by all subsequent scores meeting MID criteria compared with baseline [31].

A mechanistic population PK model was used to characterize the $\mathrm{PK}$ of abemaciclib in the MONARCH 2 population [32]. The resulting model parameter estimates were used to simulate individual patient exposure metrics, including area under the concentration-versus-time curve during one dosing interval at steady state $\left(\mathrm{AUC}_{\tau, \mathrm{ss}}\right)$, maximum concentration at steady-state $\left(\mathrm{C}_{\mathrm{max}, \mathrm{ss}}\right)$, and minimum/trough concentration at steady state $\left(\mathrm{C}_{\mathrm{min}, \mathrm{ss}}\right)$, and summarized to compare the study PK population with the Japanese PK subpopulation.

\section{Results}

\section{Patients}

The MONARCH 2 study enrolled 669 patients between August 7, 2014, and December 29, 2015, 446 and 223 of whom were allocated to receive abemaciclib plus fulvestrant or placebo plus fulvestrant, respectively. Of these, 95 patients were enrolled in Japan (abemaciclib, $n=64$; placebo, $n=31$; Online Resource 2). In the abemaciclib group, 20 patients in the Japanese subpopulation (31.3\%) initially received a $200 \mathrm{mg}$ dose before a mandatory dose reduction to $150 \mathrm{mg}$ (in comparison with $27.4 \%$ of the overall population [13]). At the time of data cutoff for the final PFS analysis, $30(46.9 \%)$ and $8(25.8 \%)$ patients in the abemaciclib and placebo groups of the Japanese subpopulation, respectively, were still on-treatment. The reason for discontinuation of study drug was most frequently PD (abemaciclib: $n=27$, 42.2\%; placebo: $n=23,74.2 \%$ ).

Table 1 summarizes demographic and baseline clinical characteristics by treatment group. Across treatment groups in the Japanese subpopulation, the majority of patients (60.0\%) were post-menopausal with a median age of 58.0 (min-max, 32.0-81.0) years. Approximately half (50.5\%) had visceral disease, and the majority had secondary ET resistance ( $\geq 71 \%$ ), Eastern Cooperative Oncology Group performance status (ECOG PS) scores of $0(\geq 84 \%)$, and progesterone receptor-positive tumors $(>82 \%)$. Although the Japanese subpopulation was generally comparable to the overall MONARCH 2 population for most baseline characteristics, a lower proportion of patients in the Japanese subpopulation were post-menopausal $(60.0 \%)$ compared with the overall population (82.4\%; Table 1 ). In addition, the Japanese subpopulation had a lower proportion of patients (9.5\%) with an ECOG PS score of 1 compared with the overall population (39.3\%). Among abemaciclib-treated patients, a lower proportion had primary ET resistance (18.8\%) and prior adjuvant chemotherapy (31.3\%) in the Japanese ITT population compared with the overall ITT population (24.9 and $46.9 \%$, respectively), resulting in an imbalance between 
Table 1 Baseline demographics and clinical characteristics

\begin{tabular}{|c|c|c|c|c|c|}
\hline \multirow[t]{2}{*}{ Characteristic } & & \multicolumn{2}{|c|}{$\begin{array}{l}\text { Japanese ITT population } \\
(N=95)\end{array}$} & \multicolumn{2}{|c|}{$\begin{array}{l}\text { Overall ITT population } \\
(N=669)\end{array}$} \\
\hline & & $\begin{array}{l}\text { Abemaciclib }+ \text { ful- } \\
\text { vestrant }(n=64)\end{array}$ & $\begin{array}{l}\text { Placebo + fulves- } \\
\text { trant }(n=31)\end{array}$ & $\begin{array}{l}\text { Abemaciclib + ful- } \\
\text { vestrant }(n=446)\end{array}$ & $\begin{array}{l}\text { Placebo + ful- } \\
\text { vestrant } \\
(n=223)\end{array}$ \\
\hline Age, years & Median (range) & $56.5(32-76)$ & $58.0(32-81)$ & $59.0(32-91)$ & $62.0(32-87)$ \\
\hline Metastatic site, $n(\%)$ & $\begin{array}{l}\text { Visceral } \\
\text { Bone only } \\
\text { Other }\end{array}$ & $\begin{array}{l}35(54.7) \\
11(17.2) \\
18(28.1)\end{array}$ & $\begin{array}{l}13(41.9) \\
8(25.8) \\
10(32.3)\end{array}$ & $\begin{array}{l}245(54.9) \\
123(27.6) \\
75(16.8)\end{array}$ & $\begin{array}{l}128(57.4) \\
57(25.6) \\
38(17.0)\end{array}$ \\
\hline Menopausal status, $n(\%)$ & $\begin{array}{l}\text { Pre/peri- } \\
\text { Post }\end{array}$ & $\begin{array}{l}26(40.6) \\
38(59.4)\end{array}$ & $\begin{array}{l}12(38.7) \\
19(61.3)\end{array}$ & $\begin{array}{l}72(16.1) \\
371(83.2)\end{array}$ & $\begin{array}{l}42(18.8) \\
180(80.7)\end{array}$ \\
\hline $\begin{array}{l}\text { Progression while receiving neo- } \\
\text { adjuvant/adjuvant ET, } n(\%)\end{array}$ & Yes & $25(39.1)$ & $17(54.8)$ & $197(44.2)$ & $103(46.2)$ \\
\hline ET sensitivity & $\begin{array}{l}\text { Primary resistance } \\
\text { Secondary resistance }\end{array}$ & $\begin{array}{l}12(18.8) \\
52(81.3)\end{array}$ & $\begin{array}{l}9(29.0) \\
22(71.0)\end{array}$ & $\begin{array}{l}111(24.9) \\
326(73.1)\end{array}$ & $\begin{array}{l}58(26.0) \\
163(73.1)\end{array}$ \\
\hline Prior chemotherapy & $\begin{array}{l}\text { Neoadjuvant } \\
\text { Adjuvant }\end{array}$ & $\begin{array}{l}10(15.6) \\
20(31.3)\end{array}$ & $\begin{array}{l}9(29.0) \\
14(45.2)\end{array}$ & $\begin{array}{l}75(16.8) \\
209(46.9)\end{array}$ & $\begin{array}{l}40(17.9) \\
103(46.2)\end{array}$ \\
\hline ECOG PS, $n(\%)$ & $\begin{array}{l}0 \\
1\end{array}$ & $\begin{array}{l}60(93.8) \\
4(6.3)\end{array}$ & $\begin{array}{l}26(83.9) \\
5(16.1)\end{array}$ & $\begin{array}{l}264(59.2) \\
176(39.5)\end{array}$ & $\begin{array}{l}136(61.0) \\
87(39.0)\end{array}$ \\
\hline $\mathrm{PgR}, n(\%)$ & Positive & $53(82.8)$ & $27(87.1)$ & $339(76.0)$ & 171 (76.7) \\
\hline
\end{tabular}

ECOG PS Eastern Cooperative Oncology Group Performance Status, ET endocrine therapy, ITT intent-to-treat, $N$ number of patients in analysis population, $n$ number of patients in category or group, $P g R$ progesterone receptor

treatment arms only within the Japanese subpopulation (Table 1).

\section{Efficacy}

At the final PFS analysis (data cutoff date: February 14, 2017; median follow-up time 19.5 months), 53 PFS events (abemaciclib: $n=30,46.9 \%$; placebo: $n=23,74.2 \%$ ) were observed in the Japanese subpopulation. The abemaciclib group had a median PFS of 21.2 months compared with 14.3 months in the placebo group (HR: 0.672; 95\% CI: 0.380-1.189; Fig. 1). In the Japan subpopulation, compared with the control group, the abemaciclib group had a higher proportion of patients with a best response of CR or PR (ORR: abemaciclib, 37.5\%; placebo, 12.9\%; Table 2). The proportion of patients with PD was higher in the placebo group $(6.5 \%)$ compared with the abemaciclib group $(3.1 \%)$.

\section{Treatment exposure and pharmacokinetics}

Dose adjustments and exposure for the Japanese subpopulation of MONARCH 2 are summarized in Online Resource 3. Median duration of abemaciclib/placebo was 64.7 and 65.0 weeks in the abemaciclib and placebo groups, respectively. Median duration of fulvestrant was 75.0 and 65.0 weeks in the abemaciclib and placebo groups, respectively. The median dose intensity for abemaciclib was $231.2 \mathrm{mg} /$ day, and median relative dose intensity was $69.7 \%$.
The dose reduction rate and dose omission rate for abemaciclib due to an adverse event (AE) was 54.0 and $82.5 \%$, respectively (placebo, 3.2 and 19.4\%, respectively; Online Resource 3). For comparison, in the overall ITT population, the median dose intensity for abemaciclib was $273.1 \mathrm{mg} /$ day and median relative dose intensity was $79.8 \%$, with dose reduction and omission rates due to AEs 42.9 and $51.9 \%$, respectively (placebo, 1.3 and $11.7 \%$, respectively).

Plasma concentrations of abemaciclib for individual patients over the course of the analysis are shown in Fig. 2. Abemaciclib PK steady-state exposure metrics $\left(\mathrm{AUC}_{\tau, \mathrm{ss}}\right.$, $\mathrm{C}_{\text {max,ss }}, \mathrm{C}_{\text {min,ss }}$ ) in the Japanese PK subpopulation were similar to the overall MONARCH $2 \mathrm{PK}$ analysis population, with comparable inter-individual variability.

\section{Safety}

At the primary endpoint analysis (February 14, 2017), all patients in both treatment groups in the Japanese subpopulation reported at least 1 TEAE, with a higher proportion of patients in the abemaciclib group reporting grade $3(68.3 \%)$ or grade $4(6.3 \%)$ TEAEs compared with the placebo group (grade 3, 22.6\%; grade 4, $0 \%$ ). No grade 5 TEAEs were reported in the Japanese subpopulation.

The most common TEAE in both treatment groups was diarrhea, which was reported at a higher frequency in the abemaciclib group (any grade, 95.2\%; grade $\geq 3,14.3 \%$ ) compared with the placebo group (any grade, $25.8 \%$; grade $\geq 3,3.2 \%$; Table 3 ). This is similar to the frequency 
Fig. 1 Progression-free survival. PFS analysis at the February 14, 2017 data cutoff date for the MONARCH 2 Japanese subpopulation. PFS was defined as the time from the date of randomization until the date of radiographic documentation of progression, based on investigator assessment, or the date of death, whichever was earlier. The curves and medians (95\% CI) were estimated using the Kaplan-Meier method. $C I$ confidence interval, $H R$ hazard ratio, No. number, $P F S$ progression-free survival

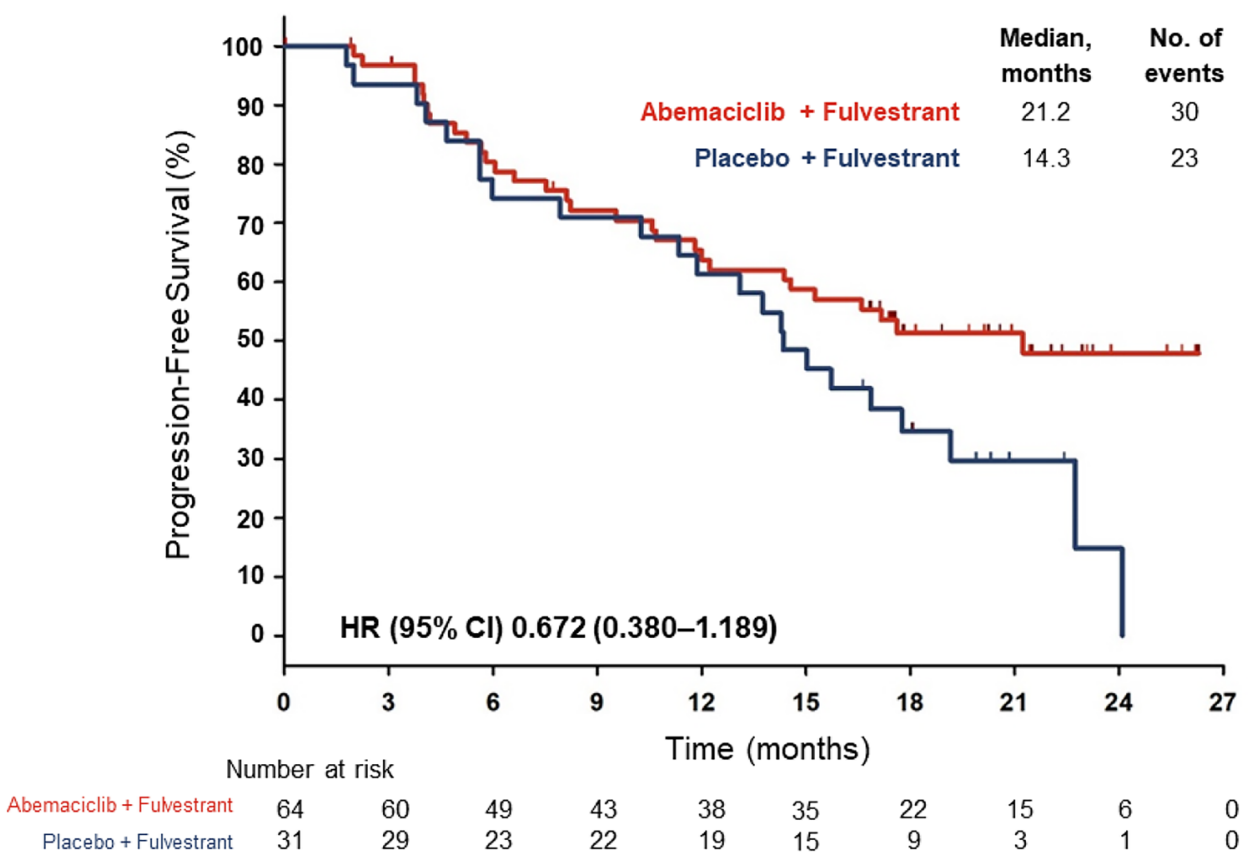

\begin{tabular}{|c|c|c|c|c|}
\hline \multirow[t]{2}{*}{ Best overall response $^{\mathrm{a}}$} & \multicolumn{2}{|c|}{$\begin{array}{l}\text { Abemaciclib + fulvestrant } \\
(N=64)\end{array}$} & \multicolumn{2}{|c|}{$\begin{array}{l}\text { Placebo + fulvestrant } \\
(N=31)\end{array}$} \\
\hline & $n(\%)$ & $95 \% \mathrm{CI}^{\mathrm{b}}$ & $n(\%)$ & $95 \% \mathrm{CI}^{\mathrm{b}}$ \\
\hline Complete response (CR) & $2(3.1)$ & $-1.1,7.4$ & $0(0.0)$ & NA \\
\hline Partial response (PR) & $22(34.4)$ & $22.7,46.0$ & $4(12.9)$ & $1.1,24.7$ \\
\hline Stable disease (SD) & $36(56.3)$ & $44.1,68.4$ & $25(80.6)$ & $66.7,94.6$ \\
\hline SD persistent for $\geq 6$ months & $26(40.6)$ & $28.6,52.7$ & $19(61.3)$ & $44.1,78.4$ \\
\hline Progressive disease (PD) & $2(3.1)$ & $-1.1,7.4$ & $2(6.5)$ & $-2.2,15.1$ \\
\hline Objective PD & $2(3.1)$ & $-1.1,7.4$ & $2(6.5)$ & $-2.2,15.1$ \\
\hline Not evaluable & $2(3.1)$ & $-1.1,7.4$ & $0(0.0)$ & NA \\
\hline Objective response rate $(\mathrm{CR}+\mathrm{PR})$ & $24(37.5)$ & $25.6,49.4$ & 4 (12.9) & $1.1,24.7$ \\
\hline Disease control rate $(\mathrm{CR}+\mathrm{PR}+\mathrm{SD})$ & $60(93.8)$ & $87.8,99.7$ & $29(93.5)$ & $84.9,102.2$ \\
\hline Clinical benefit rate $(\mathrm{CR}+\mathrm{PR}+\mathrm{SD} \geq 6$ months) & $50(78.1)$ & $68.0,88.3$ & $23(74.2)$ & $58.8,89.6$ \\
\hline
\end{tabular}

Data cutoff date: February 14, 2017

$C I$ confidence interval, $N$ number of patients in population, $n$ number of patients, $N A$ not applicable, RECIST Response Evaluation Criteria in Solid Tumors

${ }^{a}$ Response was determined by investigators for all patients whose disease was evaluable using RECIST version 1.1

${ }^{\mathrm{b}} \mathrm{CIs}$ were based on normal approximation for TEAEs of diarrhea reported in the global population (any grade, abemaciclib: 86.4\%; placebo: $24.7 \%$ [13]). The abemaciclib group also reported a higher frequency of hematologic events, including neutropenia (abemaciclib: any grade, $79.4 \%$; grade $\geq 3,44.4 \%$; placebo: any grade, $0 \%$ ); leukopenia (abemaciclib: any grade, $66.7 \%$; grade $\geq 3$, $20.6 \%$; placebo: any grade, $0 \%$ ), anemia (abemaciclib: any grade, $46.0 \%$; grade $\geq 3,9.5 \%$; placebo: any grade, $3.2 \%$; grade $\geq 3,3.2 \%$ ), and thrombocytopenia (abemaciclib: any grade, $33.3 \%$; grade $\geq 3,4.8 \%$; placebo: any grade, $0 \%$ ). TEAEs of elevated alanine aminotransferase (ALT; abemaciclib: any grade, $34.9 \%$; grade $\geq 3,9.5 \%$; placebo: any grade, $3.2 \%$; grade $\geq 3,0 \%$ ) and aspartate aminotransferase (AST; abemaciclib: any grade, $30.2 \%$; grade $\geq 3,6.3 \%$; placebo: any grade, $6.5 \%$; grade $\geq 3,0 \%$ ) were also more common in the abemaciclib group compared with placebo.

Additional TEAEs to note include fatigue, which was reported less frequently in the Japanese subpopulation 


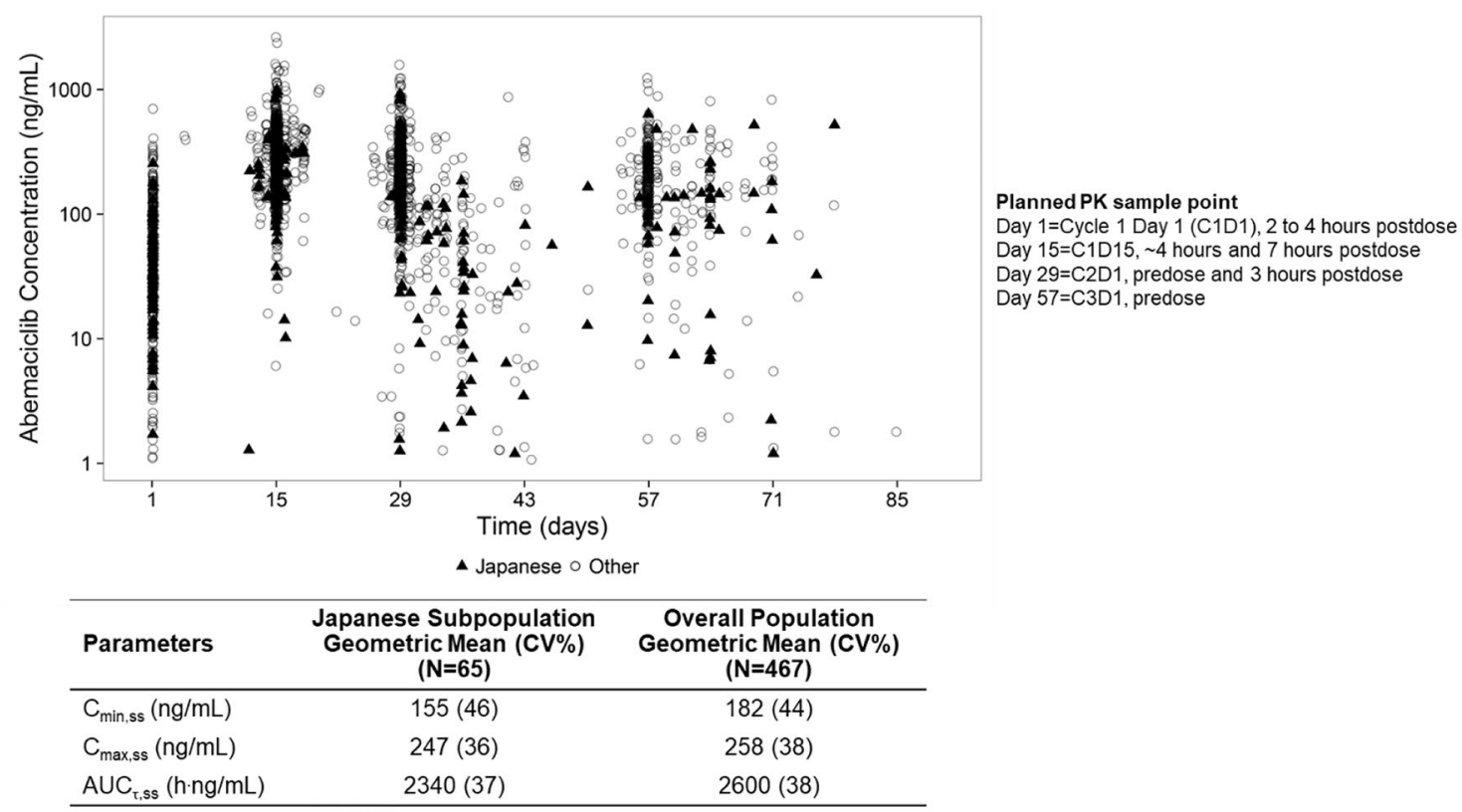

Fig. 2 Pharmacokinetic analysis of abemaciclib in patients receiving abemaciclib plus fulvestrant ${ }^{\mathrm{a}}$. Blood samples for assessment of abemaciclib concentration in plasma were obtained at the indicated prescheduled times on Days 1 and 15 of Cycle 1 and on Day 1 of Cycles 2 and 3 and measured with a validated assay. Plasma concentrations of abemaciclib for individual patients over the course of the analysis are shown in the top graph, with geometric mean trough and peak concentrations (CV\%) for the Japanese subpopulation and MONARCH 2 study population summarized in the table. ${ }^{a}$ The PK analyses are for patients receiving the 200-mg dose (pre-amendment

(abemaciclib: any grade, $11.1 \%$; grade $\geq 3,1.6 \%$; placebo: any grade, $16.1 \%$; grade $\geq 3,0 \%$ ) compared with the overall safety population (abemaciclib: any grade, $39.9 \%$; grade $\geq 3$, 2.7\%; placebo: any grade, $26.9 \%$; grade $\geq 3,0.4 \%$; [13]). In addition, one patient in the abemaciclib group in the Japanese safety population had 1 event of grade 1 pneumonitis (interstitial lung disease; ILD).

The AEs leading to abemaciclib dose adjustments were most commonly diarrhea (dose reduction: $23.8 \%$; dose omission: $22.2 \%$ ) and neutropenia (dose reduction: $12.7 \%$; dose omission: 33.3\%) (Online Resource 3), in accordance with previous findings in the overall population (dose reductions: diarrhea, 18.8\%; neutropenia, 10.0\%; dose omissions: diarrhea, $18.8 \%$; neutropenia, $16.3 \%$ ) [13]. Four (6.3\%) patients in the abemaciclib group discontinued study treatment due to an $\mathrm{AE}$ (placebo, 0\%), which included 2 events of druginduced liver injury and 1 event each of ALT and AST elevation.

\section{Quality of life}

At baseline, the EORTC QLQ-C30 global health status score and the EORTC QLQ-C30 and QLQ-BR23 functional and dose) and patients receiving the 150-mg dose (post-amendment dose) combined. PK analyses of abemaciclib were conducted on patients who had received at least 1 dose of abemaciclib and had PK samples collected, and included ET-naïve patients who were excluded from the ITT population. $A U C_{\tau, s s}$ area under the concentration versus time curve during one dosing interval at steady state, $C_{\max , s s}$ maximum concentration at steady-state, $C_{\text {min,ss }}$ minimum/trough concentration at steady state, $C V$ coefficient of variation, ET endocrine therapy, ITT intent-to-treat, $P K$ pharmacokinetics

symptom scores were generally similar between treatment groups (Table 4). Change from baseline for assessment items on the EORTC QLQ-C30 and QLQ-BR23 were not substantially different between treatment groups, except for a numerically lower diarrhea score in the placebo group which met the clinically meaningful threshold (mean [SE]: abemaciclib, 28.2 [2.1]; placebo, 2.6 [2.9]). There were no TTSD differences between treatment arms (confidence intervals cross 1) for all items except role functioning, which favored the abemaciclib group (Fig. 3a and b). The other notable exception was the diarrhea item, which numerically favored the placebo group.

\section{Overall survival, updated PFS, and updated safety}

The cutoff date for the OS analysis was June 20, 2019, with a median follow-up time of 47.7 months. The updated PFS analysis at this data cutoff was consistent with the final PFS analysis (Online Resource 4a; HR: 0.562; 95\% CI: $0.338-0.934)$. The updated median PFS was 23.8 months in the abemaciclib group compared with 14.3 months in the placebo group, reflecting a 9.5-month improvement in PFS with abemaciclib. The 36-month PFS rate was 38.6\% (95\% 
Table 3 Treatment-emergent adverse events occurring in $\geq 20 \%$ of Japanese patients by grade

\begin{tabular}{|c|c|c|c|c|c|c|}
\hline \multirow[t]{2}{*}{$\geq 20 \%$ in either group, $n(\%)$} & \multicolumn{3}{|c|}{ Abemaciclib + fulvestrant $(N=63)$} & \multicolumn{3}{|c|}{ Placebo + fulvestrant $(N=31)$} \\
\hline & All & Grade 3 & Grade 4 & All & Grade 3 & Grade 4 \\
\hline Any & $63(100)$ & $43(68.3)$ & $4(6.3)$ & $31(100)$ & $7(22.6)$ & $0(0.0)$ \\
\hline Diarrhea & $60(95.2)$ & $9(14.3)$ & $0(0.0)$ & $8(25.8)$ & $1(3.2)$ & $0(0.0)$ \\
\hline Neutropenia & $50(79.4)$ & $27(42.9)$ & $1(1.6)$ & $0(0.0)$ & $0(0.0)$ & $0(0.0)$ \\
\hline Leukopenia & $42(66.7)$ & $13(20.6)$ & $0(0.0)$ & $0(0.0)$ & $0(0.0)$ & $0(0.0)$ \\
\hline Anemia & $29(46.0)$ & $6(9.5)$ & $0(0.0)$ & $1(3.2)$ & $1(3.2)$ & $0(0.0)$ \\
\hline Nausea & $23(36.5)$ & $3(4.8)$ & $0(0.0)$ & $7(22.6)$ & $1(3.2)$ & $0(0.0)$ \\
\hline ALT increased & $22(34.9)$ & $5(7.9)$ & $1(1.6)$ & $1(3.2)$ & $0(0.0)$ & $0(0.0)$ \\
\hline Thrombocytopenia & $21(33.3)$ & $2(3.2)$ & $1(1.6)$ & $0(0.0)$ & $0(0.0)$ & $0(0.0)$ \\
\hline Abdominal pain & $20(31.7)$ & $0(0.0)$ & $0(0.0)$ & $5(16.1)$ & $0(0.0)$ & $0(0.0)$ \\
\hline AST increased & $19(30.2)$ & $4(6.3)$ & $0(0.0)$ & $2(6.5)$ & $0(0.0)$ & $0(0.0)$ \\
\hline Dysgeusia & $18(28.6)$ & $0(0.0)$ & $0(0.0)$ & $1(3.2)$ & $0(0.0)$ & $0(0.0)$ \\
\hline Stomatitis & $18(28.6)$ & $1(1.6)$ & $0(0.0)$ & $7(22.6)$ & $0(0.0)$ & $0(0.0)$ \\
\hline Vomiting & $17(27.0)$ & $1(1.6)$ & $0(0.0)$ & $3(9.7)$ & $0(0.0)$ & $0(0.0)$ \\
\hline Blood creatinine increased & $15(23.8)$ & $0(0.0)$ & $0(0.0)$ & $0(0.0)$ & $0(0.0)$ & $0(0.0)$ \\
\hline Decreased appetite & $15(23.8)$ & $2(3.2)$ & $0(0.0)$ & $5(16.1)$ & $0(0.0)$ & $0(0.0)$ \\
\hline Pyrexia & $15(23.8)$ & $0(0.0)$ & $0(0.0)$ & $3(9.7)$ & $0(0.0)$ & $0(0.0)$ \\
\hline Rash & $15(23.8)$ & $0(0.0)$ & $0(0.0)$ & $3(9.7)$ & $0(0.0)$ & $0(0.0)$ \\
\hline Headache & $14(22.2)$ & $1(1.6)$ & $0(0.0)$ & $8(25.8)$ & $0(0.0)$ & $0(0.0)$ \\
\hline Nasopharyngitis & $10(15.9)$ & $0(0.0)$ & $0(0.0)$ & $10(32.3)$ & $1(3.2)$ & $0(0.0)$ \\
\hline
\end{tabular}

MedDRA version 19.1; CTCAE version 4. Data cutoff date: February 14, 2017

$A L T$, alanine aminotransferase, $A S T$ aspartate aminotransferase, CTCAE Common Terminology Criteria for Adverse Events, MedDRA Medical Dictionary for Regulatory Activities, $N$ number of patients in population, $n$ number of patients
CI: 26.4-50.6) in the abemaciclib group and $11.1 \%$ (95\% CI: 2.9-25.4) in the placebo group (treatment effect difference 27.5\% [95\% CI: 10.6-44.4]). Median OS (Online Resource 4b) was not reached in the abemaciclib group of the Japanese subpopulation whereas OS in the placebo group was 47.3 months (HR: 0.755; 95\% CI: 0.390-1.463).

A higher proportion of patients in the placebo group $(n=29$ of $31 ; 93.5 \%)$ received post-discontinuation chemotherapy compared with the abemaciclib group ( $n=44$ of $64 ; 68.8 \%$ ), including $>$ fivefold higher usage of CDK4 and CDK6 inhibitors as post-discontinuation therapy (placebo, 41.9\%; abemaciclib, 7.8\%). Post-discontinuation CDK4 and CDK6 inhibitor usage was lower in the overall ITT population (placebo, 17.0\%; abemaciclib, 5.8\%) [12]. The time to first post-discontinuation chemotherapy showed a trend for improvement in the abemaciclib group compared with the placebo control, with median TTC 52.3 versus 26.8 months, respectively (HR: 0.651; 95\% CI: 0.353-1.198) and median CFS 50.2 versus 26.8 months, respectively (HR: 0.609; 95\% CI: 0.344-1.076; Online Resource 5). A summary of the types of first-line postdiscontinuation therapies is included in Online Resource 6. Of the 73 patients in the Japanese subpopulation who received any post-discontinuation therapy, the first subsequent therapy was chemotherapy for 32 patients $(43.8 \%)$, single-agent ET for 28 patients (38.4\%), and everolimusbased therapy for 6 patients $(8.2 \%)$. In comparison, of the 461 patients in the overall ITT population of MONARCH 2 who received post-discontinuation therapy, 209 (45.3\%), $119(25.8 \%)$, and 80 (17.4\%) received chemotherapy, single-agent ET, and everolimus-based therapy, respectively, as the first subsequent therapy [12].

The updated safety analysis indicated similar results to the primary analysis (Online Resource 7), with the proportions of grade $\geq 3$ TEAEs little changed from the primary endpoint analysis (abemaciclib: grade 3,66.7\%; grade 4, $7.9 \%$; placebo: grade 3, 22.6\%; grade 4, 6.5\%). Diarrhea, neutropenia, and leukopenia were again the most common TEAEs and were reported at a higher frequency in the abemaciclib group compared with the placebo group (abemaciclib: diarrhea: any grade, $95.2 \%$, [grade $\geq 314.3 \%$ ]; placebo, any grade, $35.5 \%$; [grade $\geq 3,3.2 \%$ ]; abemaciclib: neutropenia: any grade, $81.0 \%$ [grade $\geq 3,52.4 \%$ ]; placebo, any grade, 0\%]; leukopenia: abemaciclib, any grade, $69.8 \%$ [grade $\geq 3,23.8 \%$ ]; placebo, any grade, $0 \%$ ). The incidence of ILD reported in the Japanese safety population treated with abemaciclib plus fulvestrant was $4.8 \%(n=3)$, including $1(1.6 \%)$ event of grade 1 and 2 events $(3.2 \%)$ of grade 2 pneumonitis. 
Table 4 Mean baseline scores and within-treatment group change from baseline: EORTC QLQ-C30 and QLQ-BR23

\begin{tabular}{|c|c|c|c|c|}
\hline \multirow[t]{2}{*}{ Assessment } & \multicolumn{2}{|l|}{$\begin{array}{l}\text { Baseline score } \\
\text { Mean (SD) }\end{array}$} & \multicolumn{2}{|l|}{$\begin{array}{l}\text { Change from baseline }{ }^{\mathrm{a}} \\
\text { Least squares mean (SE) }\end{array}$} \\
\hline & $\begin{array}{l}\text { Abemaciclib + fulves- } \\
\text { trant }(n=62)\end{array}$ & $\begin{array}{l}\text { Placebo + fulvestrant } \\
(n=31)\end{array}$ & $\begin{array}{l}\text { Abemaciclib + fulves- } \\
\text { trant }(n=62)\end{array}$ & $\begin{array}{l}\text { Placebo + fulvestrant } \\
(n=31)\end{array}$ \\
\hline \multicolumn{5}{|l|}{ EORTC QLQ-C30 } \\
\hline Global health status & $70.0(20.3)$ & $67.7(23.6)$ & $-5.4(1.8)$ & $-5.6(2.5)$ \\
\hline \multicolumn{5}{|l|}{ Functional scales } \\
\hline Physical & $82.8(19.1)$ & $84.3(19.4)$ & $-0.20(1.3)$ & $-3.1(1.8)$ \\
\hline Role & $83.3(23.0)$ & $85.0(25.9)$ & $-2.8(1.8)$ & $-7.9(2.5)$ \\
\hline Emotional & $76.4(20.0)$ & $79.8(18.6)$ & $6.1(1.2)$ & $4.9(1.7)$ \\
\hline Cognitive & $83.6(18.2)$ & $87.1(15.3)$ & $-1.9(1.6)$ & $-3.4(2.2)$ \\
\hline Social & $85.5(23.5)$ & $85.0(21.7)$ & $0.4(1.6)$ & $0.7(2.2)$ \\
\hline \multicolumn{5}{|l|}{ Symptom scales } \\
\hline Fatigue & $25.1(19.0)$ & $26.5(21.8)$ & $5.1(1.8)$ & $6.6(2.5)$ \\
\hline Nausea and vomiting & $2.2(9.3)$ & $2.2(5.7)$ & $2.5(0.8)$ & $1.6(1.1)$ \\
\hline Pain & $23.1(24.0)$ & $25.3(25.8)$ & $-2.8(1.7)$ & $4.1(2.4)$ \\
\hline Dyspnea & $12.4(17.3)$ & $16.1(24.1)$ & $4.8(1.7)$ & $-0.3(2.4)$ \\
\hline Insomnia & $14.0(18.6)$ & $22.6(30.3)$ & $0.6(2.1)$ & $3.9(2.9)$ \\
\hline Appetite loss & $10.2(18.7)$ & $11.8(20.3)$ & $3.8(1.7)$ & $1.1(2.3)$ \\
\hline Constipation & $8.6(18.0)$ & $14.0(18.8)$ & $3.4(1.8)$ & $0.9(2.5)$ \\
\hline Diarrhea & $6.5(13.3)$ & $4.3(11.4)$ & $28.2(2.1)$ & $2.6(2.9)$ \\
\hline Financial difficulties & $14.0(26.0)$ & $6.5(15.9)$ & $-2.6(1.2)$ & $-0.5(1.7)$ \\
\hline \multicolumn{5}{|c|}{ EORTC QLQ-BR23 ${ }^{\mathrm{b}}$ Functional scales } \\
\hline Body image & $71.4(22.9)$ & $79.6(21.7)$ & $0.9(1.8)$ & $-1.2(2.5)$ \\
\hline Sexual functioning & $3.5(9.6)$ & $6.5(12.7)$ & $-0.8(0.7)$ & $0.1(1.0)$ \\
\hline Future perspectives & $42.5(29.1)$ & $43.0(28.8)$ & $15.9(2.3)$ & $17.6(3.2)$ \\
\hline \multicolumn{5}{|l|}{ Symptom scales } \\
\hline Systemic therapy side effects & $13.7(11.2)$ & $14.6(10.6)$ & $5.8(0.9)$ & $2.1(1.3)$ \\
\hline Breast & $16.9(20.1)$ & $20.4(21.7)$ & $-4.7(1.3)$ & $-1.7(1.8)$ \\
\hline Arm & $15.9(16.8)$ & $17.6(19.4)$ & $-0.8(1.5)$ & $1.7(2.1)$ \\
\hline
\end{tabular}

Data cutoff date: February 14, 2017

EORTC European Organization for Research and Treatment of Cancer, MMRM mixed model-repeated measures, $n$ number of subjects in the population with baseline and post-baseline value for the question at the specified visit, $Q L Q-B R 23$ Quality of Life Questionnaire-Breast subscale, 23 items, $Q L Q$-C30 Quality of Life Questionnaire-Core 30, SD standard deviation, $S E$ standard error

${ }^{a}$ Change from baseline was assessed with a Type 3 sums of squares MMRM model (Change from Baseline $=$ Treatment + Visit + Treatment*Visit + Baseline), including all cycles for which at least $25 \%$ of patients in each group have an assessment for each of the functional and symptom scales. Unstructured covariance structure was used for the MMRM model

${ }^{b}$ Deterioration of symptoms is represented by an increase in scores; deterioration of global health status and functioning scores is represented by a decrease in scores

\section{Discussion}

To gain a better understanding of the efficacy and safety of abemaciclib in Japanese breast cancer patients, the current analysis examined the Japanese subpopulation of MONARCH 2, a phase 3 study of abemaciclib plus fulvestrant in a global population of patients with HR+, HER 2- ABC who had progressed on prior ET [12, 13, 23]. Collectively, our results indicate that in the setting of advanced, ET-resistant, HR+, HER2- breast cancer, Japanese patients derived benefit from the addition of abemaciclib to fulvestrant, with outcomes broadly consistent with those of the overall ITT population $[12,13]$. At the time of the final PFS analysis, the addition of abemaciclib to fulvestrant resulted in improvement in median PFS by 6.9 months in the Japanese subpopulation (HR: 0.672; 95\% CI: 0.380-1.189; abemaciclib, 21.2 months versus placebo, 14.3 months) whereas abemaciclib resulted in a 7.1-month improvement in median PFS in the overall ITT population (HR: 0.553 ; 95\% CI: 0.449-0.681; abemaciclib, 16.4 months versus placebo, 9.3 months) [13]. At the time of the OS analysis, which occurred 27 months 
a

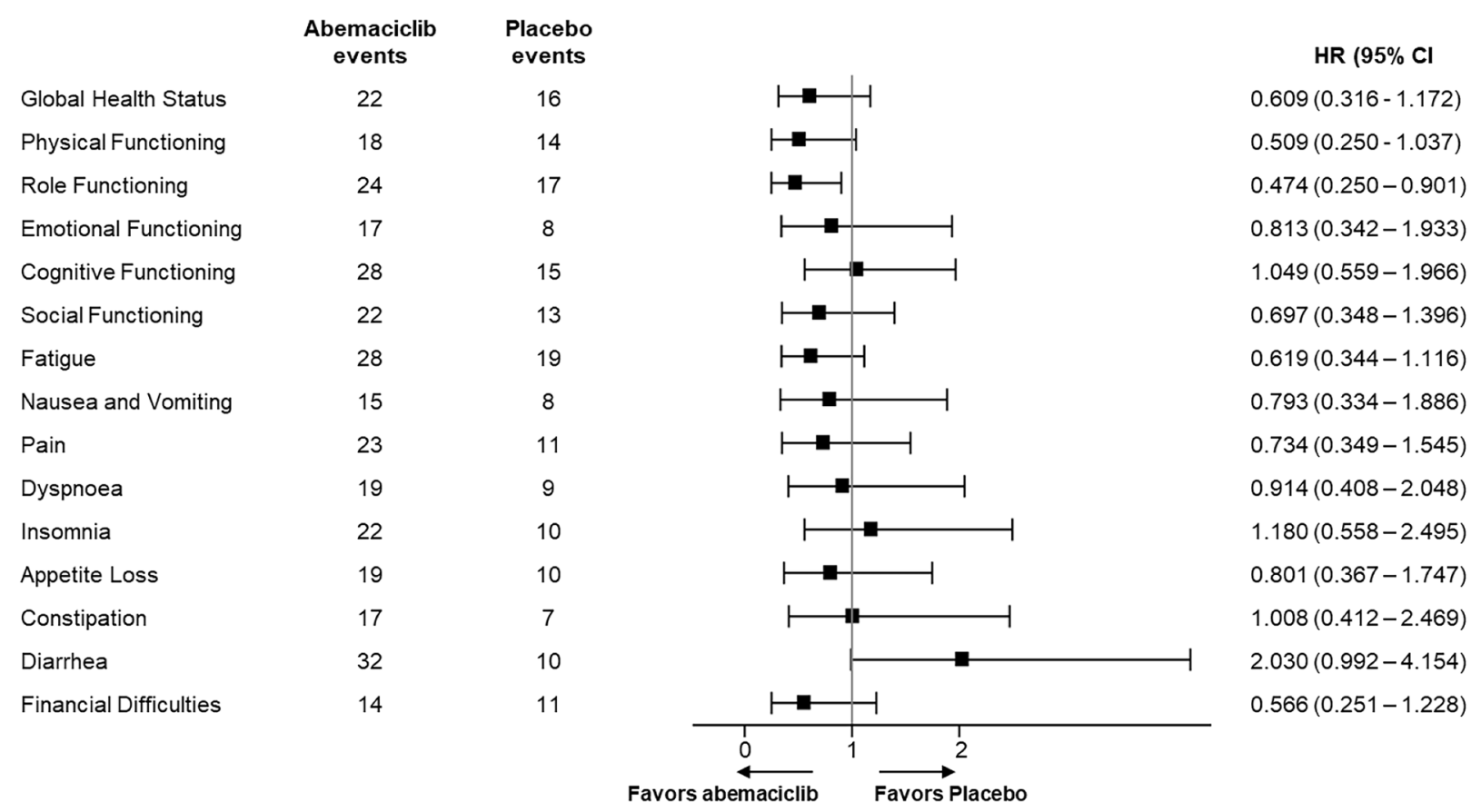

b

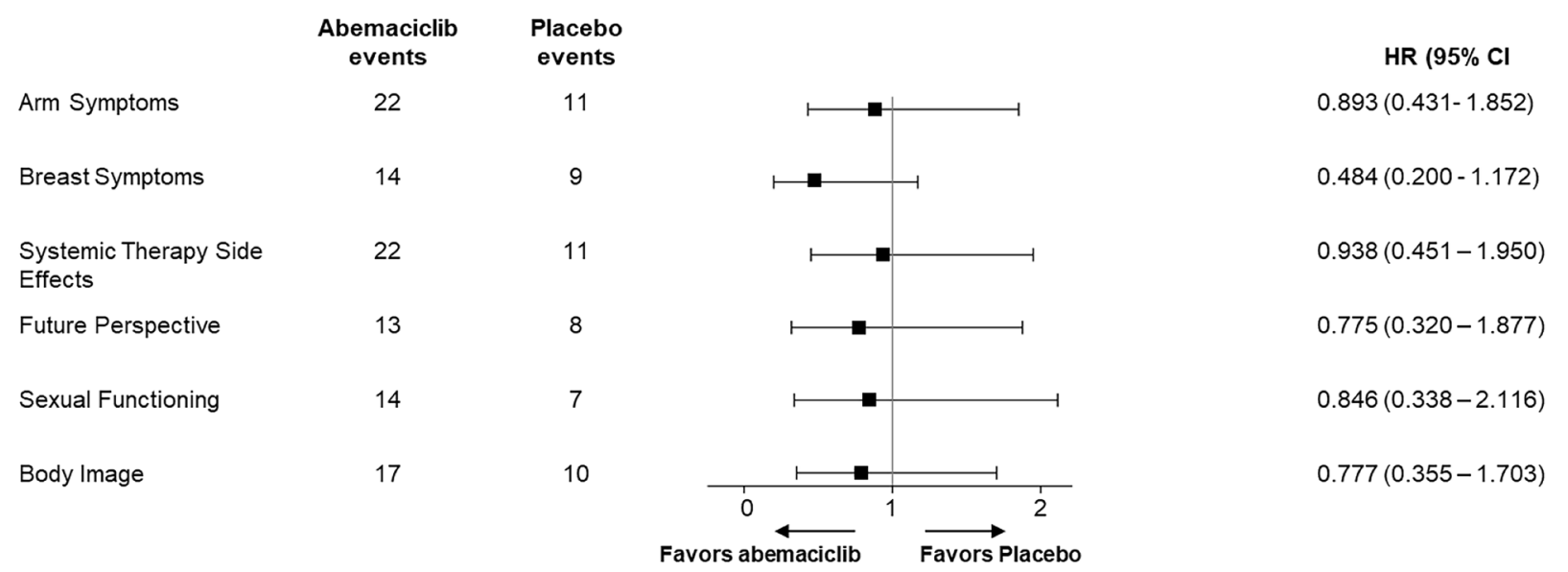

Fig. 3 Forest plots of time to sustained deterioration of individual scales on the EORTC QLQ-C30 and QLQ-BR23. a EORTC QLQC30; and b EORTC QLQ-BR23 assessments in the Japanese subpopulation of MONARCH 2. In (a), deterioration of symptoms represents an increase in scores of $\geq 10$; deterioration of global health status and functioning scores represents a decrease in scores of $\geq 10$. In (b), deterioration of symptoms of Body Image, Sexual Functioning, and Future Perspectives represents a decrease in scores

following the final PFS analysis, an updated assessment showed a 9.5-month improvement in median PFS with abemaciclib in the Japanese subpopulation (HR: 0.562; 95\% CI: 0.338-0.934; overall population: HR: 0.536; 95\% CI: $0.445-0.645$ [12]) and a $27.5 \%$ treatment effect difference in PFS rates at 36 months. The OS HR for the Japanese subpopulation (HR: 0.755 ; 95\% CI: $0.390-1.463$ ) was of $\geq 10$; deterioration of symptoms of Systemic Therapy side effects, Arm, and Breast represents an increase in score of $\geq 10$. Death was included as a deterioration event and follow-up improvement was taken into consideration. Data cutoff date: February 14, 2017. CI confidence interval, EORTC European Organization for Research and Treatment of Cancer, $H R$ hazard ratio, $Q L Q-B R 23$ Quality of Life Questionnaire-Breast subscale, 23 items, $Q L Q-C 30$, Quality of Life Questionnaire-Core 30

favorable and consistent with that observed in the overall ITT population (HR: 0.757; 95\% CI: 0.606-0.945) [12]. Median OS was not reached in the abemaciclib group of the Japanese subpopulation (whereas median OS was improved in the abemaciclib group by 9.4 months compared with placebo in the overall MONARCH 2 ITT population [12]), indicating that further follow-up is needed 
in the Japanese subpopulation. Nevertheless, this result is informative to understanding the effect of abemaciclib plus fulvestrant treatment in Japanese patients. TTC and CFS in the Japanese subpopulation also showed trends consistent with TTC and CFS in the overall population, indicating in both analysis populations that abemaciclib delayed the need for subsequent chemotherapy, an important outcome for patients with incurable disease, and that the initial effect of abemaciclib persists beyond the initial progression.

The PK and safety profiles of the Japanese subpopulation were also similar to those of the overall population. In the Japanese subpopulation, plasma concentrations of abemaciclib were as expected based on prior studies [33]. TEAEs were largely manageable with dose adjustments and/ or supportive therapy, and few discontinuations occurred due to TEAEs. As in the global population, diarrhea and hematologic events were the most common TEAEs in the abemaciclib group. However, some TEAEs occurred at a higher frequency in the Japanese subpopulation compared with the overall population [13], including a higher incidence of grade 2 and 3 (but not grade 4) neutropenia and leukopenia, Similar to this finding, a higher frequency of hematological toxicities was previously observed in the Japanese subpopulation of the global phase 3 study of another CDK4 and CDK6 inhibitor, palbociclib, in women with HR +, HER2$A B C$, with a higher incidence of $\geq$ grade 3 neutropenia in Japanese patients treated with palbociclib compared with the overall study population [9]. Although the pathogenesis of this finding is unknown, this may indicate a drug-class effect. Despite the higher incidence of neutropenia in the Japanese MONARCH 2 subpopulation, neutropenia was not associated with an increase in severe infection or febrile neutropenia, and there were no discontinuations due to neutropenia in the Japanese subpopulation.

This study revealed a higher incidence of increased ALT and AST, predominantly low grade, in abemaciclib-treated patients in the Japanese subpopulation. This result is in accordance with previous findings for the CDK4 and CDK6 inhibitor, ribociclib, which had higher liver toxicity in Japanese patients [34]. In the current analysis, no cases of Hy's law were observed, but one event each of elevated ALT and elevated AST led to study discontinuation, underscoring the importance of regular blood monitoring during abemaciclib treatment.

ILD is a well-recognized, potentially serious complication of many different cancer agents $[35,36]$ and a class side effect of CDK4 and CDK 6 inhibitors [37]. In MONARCH 2 , a slightly higher incidence of ILD was found in the Japanese safety population ( 3 events; $4.8 \%$ ) compared with the overall safety population (12 events; $2.7 \%$ ), indicating the need for regular monitoring of patients treated with abemaciclib for symptoms of ILD.
Global HRQoL, most symptoms, and functioning scales did not meet the threshold for clinically meaningful differences for the treatment arms. The only exception to this was the QLQ-C30 diarrhea score, which favored the placebo arm with clinically meaningful differences. There were no TTSD differences between treatment arms for global HRQoL, most symptoms (except diarrhea), or functioning (except role function). These findings are in accordance with a higher proportion of patients treated with abemaciclib reporting TEAEs of diarrhea compared with the placebo group. However, diarrhea appeared to be effectively managed by dose adjustments and supportive care, as no patients discontinued due to diarrhea in the Japanese subpopulation. Collectively, the current results demonstrate that patients treated with abemaciclib plus fulvestrant did not experience a clinically meaningful detriment in their HRQoL in terms of general health status and across multiple functional and symptom scales and are in agreement with the HRQoL findings in the overall MONARCH 2 study population [23].

These results should be considered in light of the limitations of this analysis. Notably, the sample size in the Japanese subpopulation is small, and statistical hypothesis testing was not applied to this analysis. Additional considerations include the noted differences between the Japanese subpopulation and the overall population that potentially could affect response to treatment in terms of both efficacy outcomes and tolerability, e.g., the Japanese subpopulation had a higher proportion of pre/perimenopausal women and a lower proportion of abemaciclib-treated patients with primary ET resistance and prior chemotherapy compared to the overall population. In addition, the Japanese subpopulation had higher usage of post-discontinuation CDK4 and CDK 6 inhibitors.

\section{Conclusion}

Consistent with the findings of the global study [12, 13, 23], Japanese patients in the MONARCH 2 study derived benefit from the addition of abemaciclib to fulvestrant in terms of improved PFS and delayed need for subsequent chemotherapy. In the Japanese subpopulation, abemaciclib plus fulvestrant had a manageable safety profile, without clinically meaningful differences from placebo plus fulvestrant across most HRQoL dimensions evaluated.

Supplementary Information The online version contains supplementary material available at https://doi.org/10.1007/s12282-021-01239-8.

Acknowledgements We thank the patients and their families for participating in the MONARCH 2 trial worldwide. We also thank the investigators, their support staff, and the MONARCH 2 steering committee who generously participated in this work. Medical writing (Kaye 
Stenvers, $\mathrm{PhD}$ ) and editing assistance (Antonia Baldo) were provided by Syneos Health.

Funding This study and manuscript were sponsored by Eli Lilly Japan K.K. Fulvestrant (Faslodex ${ }^{\circledR}$ ) was provided by AstraZeneca for the MONARCH 2 trial.

\section{Declarationss}

Conflict of interest Kenichi Inoue reports personal fees and grants to institution from Chugai Pharmaceutical Co., Ltd., Eisai Co., Ltd., Eli Lilly Japan K.K., and Pfizer Japan; and grants to institution from AstraZeneca, Bayer, MSD, Novartis Pharma K.K., Parexel/Puma Biotechnology, and Taiho Pharmaceutical Co., Ltd. Norikazu Masuda reports personal fees, grants, and other support (research funding to institution) from Chugai Pharmaceutical Co., Ltd. and Eisai Co., Ltd.; personal fees and other support (research funding to institution) from AstraZeneca, Eli Lilly Japan K.K., Pfizer Japan, Inc., Daiichi Sankyo Co., Kyowa Kirin Co. Ltd., Novartis Pharma K.K., and Takeda Pharmaceutical Company, Ltd; and other support (research funding to institution) from MSD and Nippon-Kayaku. Hiroji Iwata reports personal fees and grants from AstraZeneca, Chugai Pharmaceutical Co., Ltd, Daiichi Sankyo Co. Ltd., Eli Lilly Japan K.K., Kyowa Kirin Co. Ltd., Novartis Pharma K.K., and Pfizer Japan, Inc.; grants from Bayer, Boehringer Ingelheim, MSD, Nippon Kayaku, and Sanofi, and personal fees from Eisai Co., Ltd. Masato Takahashi reports personal fees from Astra Zeneca, Eisai Co., Ltd., Eli Lilly Japan K.K., and Pfizer Japan, Inc. Yoshinori Ito reports grants from A2 Healthcare, AstraZeneca, Covance, Chugai Pharmaceutical Co., Ltd, Daiichi Sankyo Co., Ltd., Eisai Co., Ltd., Eli Lilly Japan K.K., EPS International Holdings Co., Ltd., Kyowa Kirin Co., Ltd., MSD, Novartis Pharma K.K., Parexel, QVIA Services Japan K.K., and Taiho Pharmaceutical Co., Ltd. Yasuo Miyoshi reports personal fees and grants from AstraZeneca, Chugai Pharmaceutical Co., Ltd., Eisai Co., Ltd., Eli Lilly Japan K.K., Kyowa Kirin Co., Ltd., MSD, Pfizer Japan, Inc., and Taiho Pharmaceutical Co., Ltd. Takahiro Nakayama reports personal fees from AstraZeneca, Chugai Pharmaceutical Co., Ltd, Daiichi Sankyo Co., Ltd., Eisai Co., Ltd., Eli Lilly Japan K.K., Novartis Pharma K.K., and Taiho Pharmaceutical Co., Ltd. Hirofumi Mukai reports personal fees and grants from Daiichi Sankyo Co., Ltd. and Pfizer Japan, Inc.; research grants from the Japanese government; personal fees from Taiho Pharmaceutical Co., Ltd., and Takeda Pharmaceutical Company Ltd.; and membership on the Board of Directors of the Japan Breast Cancer Society. Antonio Llombart-Cussac reports grants, personal fees, and non-financial support from AstraZeneca, Eli Lilly and Company, Novartis, Pfizer, and Roche; grants and non-financial support from Eisai Co., Ltd.; grants and personal fees from Genomic Health, Inc. and GSK Tesaro; personal fees and non-financial support from Bristol; personal fees from MSD; and stock, patents, and intellectual property with MedSIR. George W. Sledge, Jr. reports personal fees from Syndax and Verseau, Inc.; grants from Pfizer; and other (board of directors) from Tessa Therapeutics. Masakazu Toi reports personal fees, grants, and other role or support from Daiichi Sankyo Co. Ltd. and Kyowa Kirin Co. Ltd.; personal fees and grants from AstraZeneca, C\&C Research Laboratories, Chugai Pharmaceutical Co., Ltd., Eisai Co., Ltd., Nippon Kayaku, Pfizer Japan, Inc., Shimadzu, Taiho Pharmaceutical Co., Ltd., Takeda Pharmaceutical Company, Ltd., and Yakult; grants and other role or support from Luxonus, Inc.; personal fees and other role or support from Eli Lilly Japan K.K. and Konica Minolta, Inc.; grants from AFI Technologies, Astellas Pharma, Inc., GL Sciences, the Japanese Breast Cancer Research Group Association, and Shionogi; personal fees from Exact Science, Genomic Health, Inc., MSD, and
Novartis Pharma K.K.; other role or support from Athenex Oncology, Bertis, Inc., BMS, Terumo Corporation, and Kansai Medical Net; and membership on the Board of Directors of the Japanese Breast Cancer Research Group Association, Organisation for Oncology and Translational Research, and Kyoto Breast Cancer Research Network. Joji Mori, Sachi Sakaguchi, Tsutomu Kawaguchi, and Yoshinori Tanizawa are employees and minor shareholders of Eli Lilly Japan K.K. JanStefan van der Walt is an employee and minor shareholder of Eli Lilly and Company, UK.

Ethical approval All the procedures performed in studies involving human participants were in accordance with the ethical standards of the institutional and/or national research committee and with the 1964 Helsinki Declaration and its later amendments or comparable ethical standards.

Informed consent Informed consent was obtained from all individual participants included in the study.

Open Access This article is licensed under a Creative Commons Attribution 4.0 International License, which permits use, sharing, adaptation, distribution and reproduction in any medium or format, as long as you give appropriate credit to the original author(s) and the source, provide a link to the Creative Commons licence, and indicate if changes were made. The images or other third party material in this article are included in the article's Creative Commons licence, unless indicated otherwise in a credit line to the material. If material is not included in the article's Creative Commons licence and your intended use is not permitted by statutory regulation or exceeds the permitted use, you will need to obtain permission directly from the copyright holder. To view a copy of this licence, visit http://creativecommons.org/licenses/by/4.0/.

\section{References}

1. GLOBOCAN Breast Cancer fact sheet. In.; 2018. https://gco.iarc. fr/today/data/pdf/fact-sheets/cancers/cancer-fact-sheets-15.pdf

2. Milani A, Geuna E, Mittica G, Valabrega G. Overcoming endocrine resistance in metastatic breast cancer: current evidence and future directions. World J Clin Oncol. 2014;5:990-1001.

3. Rugo HS, Rumble RB, Macrae E, Barton DL, Connolly HK, Dickler MN, et al. Endocrine therapy for hormone receptor-positive metastatic breast cancer: American Society of Clinical Oncology Guideline. J Clin Oncol. 2016;34:3069-103.

4. Fedele P, Sanna V, Fancellu A, Cinieri S. A clinical evaluation of treatments that target cell cycle machinery in breast cancer. Expert Opin Pharmacother. 2019;20:2305-15.

5. Finn RS, Martin M, Rugo HS, Jones S, Im SA, Gelmon K, et al. Palbociclib and letrozole in advanced breast cancer. N Engl J Med. 2016;375:1925-36.

6. Goetz MP, Toi M, Campone M, Sohn J, Paluch-Shimon S, Huober J, et al. MONARCH 3: abemaciclib as initial therapy for advanced breast cancer. J Clin Oncol. 2017;35:3638-46.

7. Hortobagyi GN, Stemmer SM, Burris HA, Yap YS, Sonke GS, Paluch-Shimon S, et al. Ribociclib as first-line therapy for HR-positive, advanced breast cancer. N Engl J Med. 2016;375:1738-48.

8. Im SA, Lu YS, Bardia A, Harbeck N, Colleoni M, Franke F, et al. Overall survival with ribociclib plus endocrine therapy in breast cancer. N Engl J Med. 2019;381:307-16.

9. Mukai H, Shimizu C, Masuda N, Ohtani S, Ohno S, Takahashi M, et al. Palbociclib in combination with letrozole in patients with 
estrogen receptor-positive, human epidermal growth factor receptor 2-negative advanced breast cancer: PALOMA-2 subgroup analysis of Japanese patients. Int J Clin Oncol. 2019;24:274-87.

10. Slamon DJ, Neven P, Chia S, Fasching PA, De Laurentiis M, Im SA, et al. Overall survival with ribociclib plus fulvestrant in advanced breast cancer. N Engl J Med. 2020;382:514-24.

11. Slamon DJ, Neven P, Chia S, Fasching PA, De Laurentiis M, Im SA, et al. Phase III randomized study of ribociclib and fulvestrant in hormone receptor-positive, human epidermal growth factor receptor 2-negative advanced breast cancer: MONALEESA-3. J Clin Oncol. 2018;36:2465-72.

12. Sledge GW Jr, Toi M, Neven P, Sohn J, Inoue K, Pivot X, et al. The effect of abemaciclib plus fulvestrant on overall survival in hormone receptor-positive, ERBB2-negative breast cancer that progressed on endocrine therapy-MONARCH 2: a randomized clinical trial. JAMA Oncol. 2019;6:116-24.

13. Sledge GW Jr, Toi M, Neven P, Sohn J, Inoue K, Pivot X, et al. MONARCH 2: abemaciclib in combination with fulvestrant in women with HR+/HER2- advanced breast cancer who had progressed while receiving endocrine therapy. J Clin Oncol. 2017;35:2875-84.

14. Turner NC, Ro J, Andre F, Loi S, Verma S, Iwata H, et al. Palbociclib in hormone-receptor-positive advanced breast cancer. $\mathrm{N}$ Engl J Med. 2015;373:209-19.

15. Turner NC, Slamon DJ, Ro J, Bondarenko I, Im SA, Masuda N, et al. Overall survival with palbociclib and fulvestrant in advanced breast cancer. N Engl J Med. 2018;379:1926-36.

16. Spring LM, Wander SA, Andre F, Moy B, Turner NC, Bardia A. Cyclin-dependent kinase 4 and 6 inhibitors for hormone receptor-positive breast cancer: past, present, and future. Lancet. 2020;395:817-27.

17. Dickler MN, Tolaney SM, Rugo HS, Cortes J, Dieras V, Patt D, et al. MONARCH 1, a phase II study of abemaciclib, a CDK4 and CDK6 inhibitor, as a single agent, in patients with refractory HR(+)/HER2(-) metastatic breast cancer. Clin Cancer Res. 2017;23:5218-24.

18. Gelbert LM, Cai S, Lin X, Sanchez-Martinez C, Del Prado M, Lallena MJ, et al. Preclinical characterization of the CDK4/6 inhibitor LY2835219: in-vivo cell cycle-dependent/independent anti-tumor activities alone/in combination with gemcitabine. Invest New Drugs. 2014;32:825-37.

19. Patnaik A, Rosen LS, Tolaney SM, Tolcher AW, Goldman JW, Gandhi L, et al. Efficacy and safety of abemaciclib, an inhibitor of CDK4 and CDK6, for patients with breast cancer, nonsmall cell lung cancer, and other solid tumors. Cancer Discov. 2016;6:740-53.

20. Torres-Guzman R, Calsina B, Hermoso A, Baquero C, Alvarez B, Amat J, et al. Preclinical characterization of abemaciclib in hormone receptor positive breast cancer. Oncotarget. 2017;8:69493-507.

21. Yap YS, Lu YS, Tamura K, Lee JE, Ko EY, Park YH, et al. Insights into breast cancer in the East vs the West: a review. JAMA Oncol. 2019. https://doi.org/10.1001/jamaoncol.2019. 0620.

22. Lee KWC, Lord S, Finn RS, Lim E, Martin A, Loi S, et al. The impact of ethnicity on efficacy and toxicity of cyclin D kinase 4/6 inhibitors in advanced breast cancer: a meta-analysis. Breast Cancer Res Treat. 2019;174:271-8.

23. Kaufman PA, Toi M, Neven P, Sohn J, Grischke EM, Andre V, et al. Health-related quality of life in MONARCH 2: abemaciclib plus fulvestrant in hormone receptor-positive, HER2-negative advanced breast cancer after endocrine therapy. Oncologist. 2020;25:e243-51.

24. Cardoso F, Costa A, Senkus E, Aapro M, Andre F, Barrios CH, et al. 3rd ESO-ESMO international consensus guidelines for advanced breast cancer (ABC 3). Ann Oncol. 2017;28:3111.

25. Cardoso F, Costa A, Senkus E, Aapro M, Andre F, Barrios CH, et al. 3rd ESO-ESMO international consensus guidelines for advanced breast cancer (ABC 3). Ann Oncol. 2017;28(1):16-33.

26. Eisenhauer EA, Therasse P, Bogaerts J, Schwartz LH, Sargent D, Ford R, et al. New response evaluation criteria in solid tumours: revised RECIST guideline (version 1.1). Eur J Cancer. 2009;45:228-47.

27. Aaronson NK, Ahmedzai S, Bergman B, Bullinger M, Cull A, Duez NJ, et al. The European Organization for Research and Treatment of Cancer QLQ-C30: a quality-of-life instrument for use in international clinical trials in oncology. J Natl Cancer Inst. 1993;85:365-76.

28. Sprangers MA, Groenvold M, Arraras JI, Franklin J, te Velde A, Muller M, et al. The European Organization for Research and Treatment of Cancer breast cancer-specific quality-of-life questionnaire module: first results from a three-country field study. $\mathbf{J}$ Clin Oncol. 1996;14:2756-68.

29. Kaplan EL, Meier P. Nonparametric estimation from incomplete observations. J Am Stat Assoc. 1958;53:457-81.

30. Osoba D, Rodrigues G, Myles J, Zee B, Pater J. Interpreting the significance of changes in health-related quality-of-life scores. J Clin Oncol. 1998;16:139-44.

31. Verma S, O'Shaughnessy J, Burris HA, Campone M, Alba E, Chandiwana D, et al. Health-related quality of life of postmenopausal women with hormone receptor-positive, human epidermal growth factor receptor 2-negative advanced breast cancer treated with ribociclib + letrozole: results from MONALEESA-2. Breast Cancer Res Treat. 2018;170:535-45.

32. Chigutsa E, Kambhampati SRP, Sykes AK, Posada MM, van der Walt JS, Turner PK. Development and application of a mechanistic population modeling approach to describe abemaciclib pharmacokinetics. CPT Pharmacometrics Syst Pharmacol. 2020;9:523-33.

33. Tate SC, Sykes AK, Kulanthaivel P, Chan EM, Turner PK, Cronier DM. A population pharmacokinetic and pharmacodynamic analysis of abemaciclib in a phase I clinical trial in cancer patients. Clin Pharmacokinet. 2018;57:335-44.

34. Yap YS, Chiu J, Ito Y, Ishikawa T, Aruga T, Kim SJ, et al. Ribociclib, a CDK 4/6 inhibitor, plus endocrine therapy in Asian women with advanced breast cancer. Cancer Sci. 2020;111:3313-26.

35. Azuma A. Kudo S [Drug-induced pneumonia and Japanese]. Nippon Naika Gakkai Zasshi. 2007;96:1077-82.

36. Skeoch S, Weatherley N, Swift AJ, Oldroyd A, Johns C, Hayton C, et al. Drug-induced interstitial lung disease: a systematic review. J Clin Med. 2018;7:356.

37. FDA Drug Safety Communication. FDA warns about rare but severe lung inflammation with Ibrance, Kisqali, and Verzenio for breast cancer. In.; 2019: https://www.fda.gov/drugs/drug-safetyand-availability/fda-warns-about-rare-severe-lung-inflammationibrance-kisqali-and-verzenio-breast-cancer

Publisher's Note Springer Nature remains neutral with regard to jurisdictional claims in published maps and institutional affiliations. 


\section{Authors and Affiliations}

Kenichi Inoue ${ }^{1} \cdot$ Norikazu Masuda $^{2} \cdot$ Hiroji Iwata $^{3} \cdot$ Masato Takahashi $^{4}$ Yoshinori Ito ${ }^{5}$ Yasuo Miyoshi ${ }^{6}$ Takahiro Nakayama ${ }^{7} \cdot$ Hirofumi Mukai $^{8} \cdot$ Jan-Stefan van der Walt ${ }^{9}$ - Joji Mori ${ }^{10}$. Sachi Sakaguchi ${ }^{10}$. Tsutomu Kawaguchi $^{10}$. Yoshinori Tanizawa ${ }^{10}$. Antonio Llombart-Cussac ${ }^{11,12}$ • George W. Sledge Jr ${ }^{13}$. Masakazu Toi ${ }^{14}$

1 Saitama Cancer Center, Saitama, Japan

2 National Hospital Organization, Osaka National Hospital, Osaka, Japan

3 Department of Breast Oncology, Aichi Cancer Center Hospital, Nagoya, Japan

4 National Hospital Organization Hokkaido Cancer Center, Sapporo, Japan

5 The Cancer Institute Hospital, Tokyo, Japan

6 Hyogo College of Medicine, Hyogo, Japan

7 Osaka International Cancer Institute, Osaka, Japan

8 National Cancer Center Hospital East, Kashiwa, Japan
9 Eli Lilly and Company, Surrey, UK

10 Eli Lilly Japan K.K., Kobe, Japan

11 Hospital Arnau de Vilanova, Valencia, Spain

12 SOLTI Breast Cancer Research Group, Barcelona, Spain

13 Stanford University School of Medicine, Stanford, CA, USA

14 Breast Cancer Unit, Breast Surgery, Graduate School of Medicine, Kyoto University Hospital, Kyoto University, 54 Shogoin-Kawaracho, Sakyo-ku, Kyoto 606-8507, Japan 